\title{
Die protestantischen Kirchen und "Europa" nach 1945
}

\section{Kunter, Katharina}

Vandenhoeck \& Ruprecht

2020

Kunter , K 2020 , Die protestantischen Kirchen und "Europa" nach 1945 . in I Dingel , J

Kusber \& M Morawiec (eds), Die europäische Integration und die Kirchen IV : Versöhnung und Ökumene, Ethik und Recht . Veröffentlichungen des Instituts für Europäische

Geschichte Mainz , no. 123 , Vandenhoeck \& Ruprecht, Göttingen , pp. 31-41 . https://doi.org/10.13109/978366656

http://hdl.handle.net/10138/336960

https://doi.org/10.13109/9783666560439.31

unspecified

acceptedVersion

Downloaded from Helda, University of Helsinki institutional repository.

This is an electronic reprint of the original article.

This reprint may differ from the original in pagination and typographic detail.

Please cite the original version. 


\title{
Der Protestantismus und „Europa“ nach 1945
}

\author{
Katharina Kunter, Frankfurt
}

„Nichts kann dem, was damals geschehen ist, einen Sinn verleihen“, formulierte im Hinblick auf den Zweiten Weltkrieg einmal Max Kohnstamm, einer der Gründerväter der Europäischen Union (EU), ,aber der einzige Weg, zumindest Lehren daraus zu ziehen, ist der Prozess der europäischen Integration“(Kohnstamm 2008, S.144). ${ }^{1}$

Nur wenige europäische Protestanten verkörperten diese Einsicht so konsequent mit ihrem diplomatischen und politischen Einsatz für die Europäische Gemeinschaft (EG) wie der 1914 geborene Niederländer Max Kohnstamm. Der studierte Historiker wuchs nach dem Krieg zu einem der engsten Vertrauten Jean Monnets heran und gehörte zu den nachdrücklichen Verfechtern des von Monnet konzipierten Schumann-Plans, der sich 1950 für die Schaffung einer Europäischen Gemeinschaft für Kohle und Stahl (EGKS) aussprach. Mit der 1952 erfolgten Gründung der EGKS, die auch als Montanunion bezeichnet wird, übertrugen ihre Mitgliedstaaten ${ }^{2}$ erstmals einen Teil ihrer nationalen Hoheitsrechte auf eine supranationale europäische Institution. Zugleich legten sie einen ersten „Grundstein für eine weitere und vertiefte Gemeinschaft unter den Völkern“, wie es in der Präambel zum EGKS-Vertrag hieß, aus dem sich dann später die EG entwickelte. Zum ersten Präsident der Hohen Behörde der EGKS wurde Jean Monnet, zum ersten Sekretär Max Kohnstamm ernannt. Dabei gehörte Kohnstamm schon früh zu den politischen Befürwortern des Wiederaufbaus und der positiven Integration der jungen Bundesrepublik Deutschland in die sich formierende westeuropäische Gemeinschaft. Was ihm politisch rasch als alternativlos erschien, rührte jedoch persönlich an tief erschütterte, existenzielle Grundfeste, wie der unter der deutschen Besatzung im Konzentrations- und Geisellager inhaftierte Niederländer später immer wieder betonte. Zu einem Schlüsselerlebnis wurde für Kohnstamm in diesem Zusammenhang 1947 eine Reise nach Deutschland, die er als Mitglied der Deutschland-Kommission der

\footnotetext{
${ }^{1}$ Hervorgehoben und übersetzt durch Friso Wielenga, Rezension zu Anjo G. Harryvan/Jan van der Harst, Max Kohnstamm. Leven en werk van een Europeaan, Utrecht 2008 (Zitat auf S. 144), vgl. https://www.unimuenster.de/NiederlandeNet/nl-wissen/geschichte/vertiefung/europa/kohnstamm.html.

${ }^{2}$ Die Gründungsmitglieder waren Frankreich, Deutschland, Italien, Niederlande, Belgien und Luxemburg.
} 
Nederlandse Hervormde Kerk unternahm, und bei der auch die im Widerstand aktive Hebe Kohlbrugge ${ }^{3}$ dabei war:

„(...) Zwei Jahre später kam ich zum ersten Mal nach Deutschland. Mein Haß und mein Glaube an die Kollektivschuld des deutschen Volkes wurde konfrontiert mit den Trümmerhaufen deutscher Städte, mit Kindern, die in diesen Trümmern wohnten, spielten, zur Schule gingen. Allmählich wurde mir klar, daß es für mein Land, für Europa, keine Zukunft gäbe ohne den Wiederaufbau Deutschlands (...).“(Kohnstamm $1985, \mathrm{~S} .3)^{4}$

Unter den Niederländern der damaligen Zeit vertrat Kohnstamm mit dieser Einsicht zwar eine Mindermeinung. Doch im 1948 gegründeten und in Genf residierenden Ökumenischen Rat der Kirchen (ÖRK) hatte Kohnstamm Gleichgesinnte.

\section{Willem Visser ' $t$ 't Hooft und das europäische Erbe des Widerstandes}

Dreh- und Angelpunkt in Genf war der reformierte, niederländische Theologe Willem Visser 't Hooft, der 1948 auf der ersten Vollversammlung des ÖRK in Amsterdam nach der kriegsunterbrochenen Aufbauphase nun endlich offiziell zum Generalsekretär gewählt worden war. Bereits seit 1939 als Generalsekretär des ÖRK designiert, konnte er seitdem das neue, kleine Generalsekretariat und die über Genf laufenden Kontakte der Ökumenischen Bewegung nutzen, um zentrale Einzelakteure und Gruppen des europäischen Widerstandes zusammen zu bringen, sie miteinander zu vernetzen und für den gemeinsamen Aufbau einer zukünftigen europäischen Ordnung zu gewinnen. ${ }^{5}$ Visser 't Hooft ging es dabei nicht nur um

\footnotetext{
${ }^{3}$ Hebe Kohlbrugge gehörte mit Visser 't Hooft in Genf zu den Mitbegründern des Schweizer Weges, der seit 1942 geheime Informationen zwischen Widerstand, Königin und der niederländischen Exilregierung in London transportierte und dabei mit dem Widerstandsnetzwerk Dutch-Paris, das Juden über Frankreich die Flucht in die Schweiz ermöglichte, zusammenarbeitete. Nach dem Krieg wurde sie Sekretärin der DeutschlandKommission der Nederlandse Hervormde Kerk. Vgl. hierzu u.a. die Erinnerungen von Hebe Kohlbrugge, Zwei mal zwei ist fünf. Mein unberechenbares Leben seit 1914. Leipzig 2003; Willem A. Visser 't Hooft, Memoirs, Genf 1973.

${ }^{4}$ Max Kohnstamm, Die alten, bösen Lieder. Das Hin und Her um die Vorbereitungen der Reagan-Reise öffnet schon verheilte Wunden, in: Die Zeit Nr. 18 vom 26. April 1985, S.3. Auf die damalige Deutschlandreise nahm Kohnstamm auch immer wieder Bezug in veröffentlichten Interviews. Vgl. auch Geert Mak, Niederlande, München 2010, S. 194.

${ }^{5}$ Vgl. hierzu und im Folgenden ausführlicher neben den Schilderungen in Visser 't Hoofts Erinnerungen u.a. Joachim Garstecki (Hg.), Die Ökumene und der Widerstand gegen Diktaturen. Nationalsozialismus und Kommunismus als Herausforderung an die Kirchen, Stuttgart 2007; Jurjen A. Zeilstra, European Unity in Ecumenical Thinking 1937-1948, Zoetermeer 1995; Martin Greschat, Der Protestantismus und die Entstehung der Europäischen Gemeinschaft, in: Martin Greschat/Wilfried Loth (Hg.), Die Christen und die Entstehung der Europäischen Gemeinschaft, Stuttgart 1994, S. 25-S. 96; Walter Lipgens (Hg.), Documents on the History of European Integration, Bd. 2, Berlin/New York 1986, S. 699-753 (redaktionell überarbeitet auch abgedruckt in
} 
die Integration des deutschen Widerstandes in eine europäische Widerstandsbewegung - zu seinen wichtigsten Verbindungsmännern gehörten der evangelische Theologe Dietrich Bonhoeffer und der im Kreisauer Kreis für das Amt des Außenministers vorgesehene Jurist Adam von Trott -, sondern insbesondere um die Ausgestaltung der ideellen Grundlagen eines gerechten und dauerhaften Friedens in Europa.

Davon überzeugt, dass die Politik der nationalen Souveränität in Europa langfristig erneut zu Spaltungen und Krieg führen würde, warb er stattdessen vehement dafür, Europa nach dem Zweiten Weltkrieg als ein vereintes und föderales Europa aufzubauen. In diesem Prozess sprach er einem grundlegend erneuerten Christentum und den in ökumenischer Zusammengehörigkeit miteinander verbundenen Kirchen eine zentrale Rolle bei der theologischen, moralischen und gesellschaftlichen Neuorientierung Europas zu. Vertiefte Einsichten zu den Schritten und den Zielen auf dem Weg dahin hatte Visser 't Hooft seit 1939 im Kontext eines intensiv geführten ökumenischen Gesprächsprozesses um die Verantwortlichkeit der Kirche für die internationale Ordnung gewonnen. ${ }^{6}$ An diesem waren in besonderer Weise Briten und Amerikaner beteiligt und engagiert; etwa in der Arbeit der britischen Peace Aims Group, deren aus der Internationalen Missionsbewegung stammender Sekretär William Paton hier eine treibende Rolle spielte. Er legte mit seinem im Juli 1941 erschienen Buch „The Church and the New Order“ einen ökumenischen Gesprächsbeitrag vor, in dem er das Ende nationaler Autarkie in Europa als Voraussetzung einer neuen Friedensordnung und die Kirchen als Motor der moralischen Erneuerung in Europa skizzierte. Diese sollte dann allerdings nicht nur auf der Stärkung der anglo-amerikanischen Beziehungen beruhen, sondern ebenso auf deren leitenden Prinzipien von Demokratie und freiem Markt. ${ }^{7}$ Auch in den USA hatten sich seit 1939, parallel zur Peace Aims Group in Großbritannien, engagierte Ökumeniker unter dem Dach des amerikanischen Federal Council of Churches (FCC) zu einer eigenen Gruppe zusammen gefunden, der Commisssion to Study the Bases on a Just and Durable Peace. ${ }^{8}$ Sie wurde von dem späteren amerikanischen

Martin Greschat/Wilfried Loth (Hg.), Die Christen und die Entstehung der Europäischen Gemeinschaft, Stuttgart 1994, S. 13-S. 23.

${ }^{6}$ Vgl. hierzu die ausführliche Darstellungen mit Quellenbelegen u.a. bei Philip M. Coupland, Britannia, Europa and Christendom. British Christians and European Integration, London 2006; John S. Nurser, For all Peoples and All Nations: The Ecumenical Church and Human Rights, Georgetown 2005; Jurjen A. Zeilstra, European Unity in Ecumenical Thinking 1937-1948, Zoetermeer 1995.

${ }^{7}$ Vgl. ausführlicher P. M. Coupland, Britannia und J. A. Zeilstra, European Unity.

${ }^{8} \mathrm{Vgl}$. hierzu ausführlicher u.a. Martin Greschat, Der Protestantismus und die Entstehung der Europäischen Gemeinschaft, in: Martin Greschat/Wilfried Loth (Hg.), Die Christen und die Entstehung der Europäischen 
Außenminister John Forster Dulles geleitet und wollte nicht nur den amerikanischen Isolationismus aufbrechen, sondern warb auch, etwa in ihrer Schrift "The Six Pillars of Peace" von 1943, vehement für die Etablierung einer internationalen Organisation. ${ }^{9}$ Denn gerechter und dauerhafter Friede in Europa und in der Welt könne nur entstehen, wenn alle Staaten auch in Europa - etwas von ihrer Unabhängigkeit an die Vereinten Nationen (UN) abgeben würden, meinte man in dieser, vor allem aus protestantischen Laien, gebildeten Gruppe. Kurz darauf nahm Dulles im Herbst 1944 an der Vorbereitungskonferenz in Dumbarton Oaks teil und war dort an der Ausarbeitung der UN-Charta mitbeteiligt; später vertrat er dann auch als Delegierter die USA bei der Gründung der UN und bei den darauffolgenden drei UNVollversammlungen.

Doch so sehr freilich insbesondere die amerikanischen Vorschläge der Studienkommission die weiteren Zielsetzungen und Diskussionen des ÖRK unmittelbar nach seiner Gründung am Ende des Zweiten Weltkrieges prägen sollten: Visser 't Hooft hatte durchaus eine eigene, davon abweichende Nachkriegsvision von Europa. So kam ihm zum einen in den Zukunftsplänen der Briten und Amerikaner der deutsche Widerstand und die zukünftige Rolle Deutschlands zu wenig vor. Zum anderen kritisierte er die „great power“-Konzeptionen Großbritanniens und der USA, die nicht zu seinem Ideal vom europäischen Föderalismus passten. ${ }^{10}$ Denn für dieses mussten - seiner Meinung nach - eben auch die kleineren und mittelgroßen europäischen Staaten selbst in die politischen Gestaltungsprozesse mit einbezogen werden. Visser 't Hooft setzte daher neben den Kirchen und der Ökumene auch auf andere Netzwerke und Gruppierungen aus dem Umfeld des europäischen Widerstandes. Zwischen dem März und dem Juni 1944 fanden in seiner Genfer Wohnung fünf geheime Treffen der europäischen Föderalisten rund um die italienischen Antifaschisten Aliero Spinelli, Ernesto Rosso und Eugenio Colorni statt. Diese hatten bereits im Juli $1941 \mathrm{ihr}$ „Manifest von Ventotene“ verfasst, in dem sie sich als eine maßgebliche Konsequenz aus dem gescheiterten Völkerbund für einen föderalen europäischen Bundesstaat

Gemeinschaft, Stuttgart 1994, S. 25-S. 96; Walter Lipgens (Hg.), Documents on the History of European Integration, Bd. 2, Berlin/New York 1986, S. 699-753 (wie FN 5).

${ }^{9}$ Vgl. ausführlicher zur Biographie Dulles Richard H. Immerman, John Forster Dulles, Piety, Pragmatism, and Power in U.S. Foreign Policy, Washington 1998. - Der Text der "Six Pillars" findet sich u.a. in: Walter Lipgens (Hg.), Europa-Föderationspläne der Widerstandsbewegung 1940-1945, München 1968, S. 740-743.

${ }^{10}$ In seiner 2015 am Europäischen Institut in Mainz verteidigten Dissertation hat sich Jan Schubert ausführlich mit den Europavorstellungen Visser 't Hoofts beschäftigt. 
ausgesprochen hatten ${ }^{11}$, und versuchten nun zusammen mit weiteren Personen aus dem französischen, tschechischen, deutschen, polnischen, jugoslawischen, dänischen und norwegischen Widerstand in Genf, diese Gedanken zu einer EU gemeinsam weiter zu entwickeln. Visser 't Hooft war als Vertreter der niederländischen Widerstands aktiv bei der Formulierung eines dabei entstandenen europäischen Manifestes beteiligt, das sich - die späteren Grundzüge der Verfassung der EU vorwegnehmend - bereits für eine gemeinsame europäische Regierung, eine gemeinsame Armee und einen gemeinsamen Gerichtshof sowie eine permanente Repräsentanz aussprach. ${ }^{12}$ Die großen Hoffnungen freilich, die Visser 't Hooft auf ein durch den europäischen Widerstand getragenes neues Europa setzte, schwanden nach dem gescheiterten Hitler-Attentat vom 20. Juli 1944 deutlich; u.a. auch wegen des von ihm zunehmend dominanter wahrgenommenen russischen und amerikanischen (ökonomischen) Imperialismus, der für die Interessen der „second and third class powers"(Zeilstra 1995, S.127) ${ }^{13}$ keinen Raum mehr ließ. So formulierte er bei einem Gespräch mit Vertretern der Peace Aim Groups am 7. November 1944 enttäuscht: „With regard to Federation, I do not think it is a practical possibility any longer. It is nonsense to talk about a European federation now. Actually a process of federation is going on, but it will make the unity of Europe harder to get rather than easier."(Zeilstra 1995, S.126) ${ }^{14}$

\section{Der Kalte Krieg als Motor und Bremse des europäischen Integrationsgedankens}

Das Engagement für eine neue internationale Friedensordnung und die Etablierung der UN als einer die universalen Menschenrechte politisch vertretenden, internationalen Organisation, das den Kernpunkt der amerikanischen Studienkommission in der ersten Hälfte der 1940er Jahre gebildet hatte, erfuhr nach dem Kriegsende durch die 1946 erfolgte Gründung der Kommission der Kirchen für Internationale Angelegenheiten (KKIA/engI. CCIA: Commission of the Churches on International Affairs) eine gezielte institutionelle Stärkung in der Ökumenischen Bewegung. Zunächst als eine Art unabhängiger, außenpolitischer „Think

\footnotetext{
${ }^{11}$ Vgl. u.a. Visser 't Hooft, Memoirs, S. 177 ff; Rolf-Ulrich Kunze, Die ganze Kirche für die ganze Welt: Willem Adolf Visser 't Hooft und der Widerstand gegen den Nationalsozialismus, 1933-1945, in: Joachim Garstecki (Hg.), Die Ökumene und der Widerstand gegen Diktaturen. Nationalsozialismus und Kommunismus als Herausforderung an die Kirchen, Stuttgart 2007 (wie FN 5), S. 32-46.

${ }^{12} \mathrm{Vgl}$. hierzu und weiterführend Sergio Pistone, The Union of European Federalists, Mailand 2008, S. 20; George-Henri Soutou, Amt und Verantwortung: Diplomaten in Deutschland und Frankreich 1933-1945, in: Johannes Hürter/Michael Mayer (Hg.), Das Auswärtige Amt in der NS-Diktatur, Berlin 2014, S. 59-S.74, hier v.a. S. 20.

${ }^{13}$ Jurjen A. Zeilstra, European Unity, S. 127

${ }^{14}$ Ebd., S. 126
} 
Tank" der Ökumene mit zwei Büros in New York und in London und zwischen Internationalem Missionsrat und sich aufbauendem ÖRK angesiedelt, wollte die KKIA die Kirchen an die aktuellen politischen Probleme heranführen und sie zur Diskussion unter christlichen Perspektiven ermutigen. ${ }^{15}$ Bei der Gründungsvollversammlung des ÖRK in Amsterdam 1948 waren ihre Mitglieder maßgeblich für die Gestaltung und Debatte in der Sektion IV zum Thema „Die Kirche und die internationale Unordnung“ verantwortlich, wobei die Anliegen der Kommission dann besonders deutlich in dem von John Forster Dulles vorgetragenen Hauptreferat zum Ausdruck kamen. Das zweite Hauptreferat allerdings, gehalten von dem tschechischen Theologen Josef L. Hromádka, setzte dem amerikanischen Werben für eine demokratisch freie Gesellschaft nach amerikanischem Muster ein explizit antiwestliches Gegenmodell entgegen. In ihm wies Hromádka nachdrücklich auf die durch den Nationalsozialismus und den Zweiten Weltkrieg sichtbar gewordenen moralischen Verfallserscheinungen des kapitalistischen Westens hin und hob stattdessen das gesamtgesellschaftliche Erneuerungspotential durch den Sozialismus hervor. Die Frage nach der Implementierung eines eigenen, föderalen Nachkriegsmodells für Europa, mit dem sich Visser 't Hooft und die protestantischen Vertreter des Widerstandes so intensiv im Krieg beschäftigt hatten, war damit unversehens an den Rand der ökumenischen Diskussionen gerückt und fand angesichts des sich verschärfenden ideologischen Tons zwischen den beiden Supermächten Sowjetunion und USA im beginnenden Kalten Krieg zunächst keine Plattform mehr. In seinem Abschlussbericht sprach sich der ÖRK, dem amerikanischen Votum folgend, für die ausdrückliche Unterstützung der UNO, für die ökumenische Akzeptanz der Menschenrechte und der Religionsfreiheit aus; versuchte aber zugleich, vorsichtig einen eigenen Weg zwischen Ost und West zu skizzieren:

„(...) Die christliche Kirche sollte die Ideologien beider verwerfen, des Kommunismus und des Laissez-faire-Kapitalismus, und danach trachten, die Menschen von der falschen Vorstellung zu befreien, diese beiden stellten die einzige Alternative dar."(Amsterdamer Dokumente 1949, S.53) ${ }^{16}$

In eine ähnliche Richtung zielte das von Visser 't Hooft im ÖRK seit 1948 vertretene sozialethische Leitkonzept der "Responsible Society“, das die politische und wirtschaftliche Verantwortung christlicher Eliten für das Gemeinwohl und das gemeinsame Streben nach

\footnotetext{
${ }^{15} \mathrm{Vgl.} \mathrm{hierzu} \mathrm{u.a.} \mathrm{ausführlicher} \mathrm{M.} \mathrm{Greschat,} \mathrm{Protestantismus,} \mathrm{S.} 41$.

${ }^{16}$ Amsterdamer Dokumente. Berichte und Reden auf der Weltkirchenkonferenz in Amsterdam 1948; Bielefeld, 2. Auflage 1949, S. 53.
} 
einer gerechten, freien und demokratischen Gesellschaft betonte. ${ }^{17}$ Schnell erkannte Visser 't Hooft allerdings auch, dass die von ihm bevorzugte ideologische Unabhängigkeit von Ost und West für die Kirchen im Osten keine realistische Option war - und für einen Dritten Weg daher kein Raum. ${ }^{18}$ Anders sah das der Schweizer Theologe Karl Barth. Ähnlich wie Hromádka war Barth davon überzeugt, dass Europa nun nach dem Desaster der beiden Weltkriege „seine einstige politische und kulturelle und glaubensmäßige Zentralstellung in der Welt verloren“ habe und „zwischen zwei Mühlsteine“ geraten sei, die sowohl vom Westen wie auch vom Osten her die Menschheit bedrohten. Würde Europa die Kraft aufbringen, sein Versäumnis in einer eigenen und europäischen Gestalt des Sozialismus nachzuholen, oder drohe doch stärker die Gefahr eines „amerikanisierten Europa“? ${ }^{19}$ Für einen tiefgreifenden kirchlich-politischen Paradigmenwechsel und für eine neue Offenheit gegenüber Sozialismus und Sozialdemokratie plädierte auch das am 8. August 1947 in Darmstadt verabschiedete „Wort des Bruderrates der Evangelischen Kirche in Deutschland (EKD) zum politischen Weg unseres Volkes", das wesentlich von Theologen des

Dahlemitischen Flügels der Bekennenden Kirche, nämlich dem Göttinger Theologieprofessor Hans-Joachim Iwand, Karl Barth sowie den beiden evangelischen Pfarrern Martin Niemöller und Hermann Diem formuliert worden war. ${ }^{20}$ Vor dem Hintergrund des Koreakrieges, der Auseinandersetzungen um die Wiederbewaffnung und die Europäische Verteidigungsgemeinschaft war „Europa“ für diese barthianisch geprägten evangelischen Kreise vor allem ein paneuropäisch gedachtes Europa. Dieses Europa sollte sowohl der Versöhnung zwischen den ehemaligen Kriegsfeinden im Osten und im Westen Europas dienen, als sich auch bewusst über die ideologischen Machtinteressen der Sowjetunion und der USA hinwegsetzen und dem transnationalen Charakters des Christentums entsprechend neue Räume der Begegnung in Europa schaffen. Voraussetzung dafür sollte ein wiedervereinigtes, neutrales Deutschland sein, das sich als Brücke zwischen Ost und West in

\footnotetext{
${ }^{17}$ Ebd., S. 50. Zum Konzept der „Responsible Society“ siehe weiterhin Willem A. Visser 't Hooft/Joseph H. Oldham, The Church and Its Function in Society, Chicago 1937; Cornelis L. Patijn, Die "verantwortliche Gesellschaft". Ein Rückblick, in: Ökumenischer Rat der Kirchen (Hg.), Es begann in Amsterdam. 40 Jahre Ökumenischer Rat der Kirchen, Frankfurt a.M. 1989, S. 62-66.

${ }^{18}$ Darauf verweist nachdrücklich Martin Greschat, Ökumenisches Handeln der Kirchen in den Zeiten des Kalten Krieges, in: Ökumenische Rundschau 49 (2000), S. 7-25; insbesondere S. 14.

${ }^{19} \mathrm{Vgl}$. Karl Barth, Die christliche Verkündigung im heutigen Europa, München 1946, S. 9.

${ }^{20}$ Vgl. ausführlicher u.a. Günter Brakelmann, Kirche und Schuld: Das Darmstädter Wort von 1947, in: Ders., Kirche in den Konflikten ihrer Zeit, München 1981; Martin Greschat, Im Zeichen der Schuld, München 1985.
} 
Europa verstand. ${ }^{21}$ In diesem Kontext erfolgte bspw. die Gründung des Französisch-

Deutschen Bruderrates 1951, die 1952 unternommene Moskaureise Martin Niemöllers sowie der erste Besuch einer Delegation des Rates der EKD bei den Kirchen in der Tschechoslowakei im März 1955. Auch die im Januar 1959 entstandene Konferenz Europäischer Kirchen (KEK), die aus einer deutsch-niederländischen Initiative um Heinrich Held und Ernst Wilm, Präsides der rheinischen und westfälischen Landeskirche, den ehemaligen oldenburgischen Oberkirchenrat Heinz Kloppenburg sowie Egbert Emmen, Generalsekretär der Nederlandse Hervormde Kerk, hervorging, wollte i. d. S. ein gemeinsames kirchliches Forum für die Begegnung und Verständigung von Christen aus Ostund Westeuropa sein.

\section{Evangelisches Engagement im westeuropäischen Integrationsprozess}

Während ein Teil der Protestanten Deutschlands und der Ökumene auf einen Dritten Weg der Kirchen zwischen Ost und West setzte, ebnete zur gleichen Zeit der französische Außenminister Robert Schuman offiziell der politischen Westintegration Deutschlands den Weg. Sein am 8. Mai 1950 an Bundeskanzler Konrad Adenauer gerichtetes Angebot, eine gemeinsame supranationale Behörde für Kohle und Stahl zu schaffen, bestätigte Adenauers Politik der Westintegration und setzte den westeuropäischen Integrationsprozess in Gang. Schon kurz zuvor, bei einer im April 1950 von der KKIA und dem ÖRK organisierten Tagung im Ökumenischen Institut im schweizerischen Bossey, hatte André Philip, der französische Protestant, sozialistische Politiker und Wirtschaftsminister sowie enge Vertraute von Charles de Gaulle Visser 't Hooft darauf hingewiesen, dass nun auch der ÖRK dem Prozess der westeuropäischen Integration mehr Aufmerksamkeit schenken müsse. ${ }^{22}$ Visser 't Hooft unterstützte dieses Anliegen und trug mit Paul Abrecht, einem amerikanischen Theologen aus der Studienabteilung des ÖRK, dazu bei, dass im Juli 1950 erstmals eine lose an den ÖRK angebundene Gruppe aus angesehenen evangelischen Persönlichkeiten aus Politik, Wirtschaft und Kirche zusammenkam und sich i. S. der Responsible Society über aktuelle Herausforderungen im europäischen Integrationsprozess austauschte. Ziel der Gruppe war der gemeinsame internationale Austausch, der dann in der Folge in die Kirchen

\footnotetext{
${ }^{21}$ Vgl. hierzu ausführlicher Thomas Sauer, Westorientierung im deutschen Protestantismus? Vorstellungen und Tätigkeit des Kronberger Kreises, München 1999, hier v.a. S. $47 \mathrm{f}$.

${ }^{22}$ Vgl. zum Ganzen ausführlicher Lucian N. Leustean, The Ecumenical Movement and the Making oft he European Community, Oxford 2014, hier insbesondere S. 24.
} 
hineingetragen werden und zu einem stärkeren Europabewusstsein mobilisieren sollte. ${ }^{23}$ Paul Abrecht wurde zum Sekretär der Gruppe ernannt, die sich zunächst als Ecumenical Commission of European Cooperation bezeichnete, dann aber, um der Unabhängigkeit der Gruppe vom ÖRK deutlicheren Ausdruck zu verleihen, in Committee on the Christian Responsibility for European Cooperation (CCREC) umbenannt wurde. Zu ihren Mitgliedern zählten neben André Philip europäische Politiker und Intellektuelle, wie z.B. der bereits erwähnte Max Kohnstamm und der ebenfalls aus der Nederlandse Hervormde Kerk stammende Politiker Conny L. Patijn; der belgische Politiker Jean Rey, eng verbunden mit der EGKS und von 1967 bis 1970 der erste Präsident der aus Europäischer Wirtschaftsgemeinschaft, Euratom und Montan-Union fusionierten Europa-Kommission; Kenneth Grubb, der Leiter des CCIA-Büros in London; Denis de Rougemont, einer der profiliertesten Schweizer Vordenker des föderalen Europas oder auch Pierre Mahillon aus Belgien, Rechtsanwalt und Leiter des evangelischen Kirchen in Belgien. Aus der Bundesrepublik nahmen anfangs Gustav Heinemann, damals noch CDU-Innenminister und Präses der EKD-Synode, und der niedersächsische Ministerpräsident Hinrich Kopf an den Treffen der teil. Intensive und kontroverse Debatten um die Zukunft Europas bestimmten die Anfangsjahre dieser Gruppe. Auch, wenn zu ihrem Kreis bspw. mit Heinemann ein ausgesprochener Kritiker des Adenauer-Kurses, der Wiederbewaffnung der Bundesrepublik und der Europäischen Verteidigungsgemeinschaft und hierdurch der Westintegration gehörte, setzte sich die Mehrheit in der CCREC aber konsequent für die westeuropäische Einigung ein. Mit dem Abschluss der Pariser Verträge veränderte sich dann allerdings die Bedeutung der CCREC, die als Gruppe bis 1974 bestand. Ihre Rolle als ein protestantisches Laienforum, auf dem europäische Verantwortungsträger zusammen mit ökumenischen Kirchenvertretern über ihre christliche Verantwortung nachdachten, schwand. Stattdessen richteten sich ihre Mitglieder und Aktivitäten jetzt stärker an der inhaltlichen Arbeit der europäischen Institutionen in Paris, Straßburg und Brüssel aus und begannen mit gezielter protestantischer bzw. christlicher Lobbyarbeit. ${ }^{24}$ Nach einer Tagung des ÖRK in der Evangelischen Akademie Arnoldshain zum Schutz der Rechte von Wanderarbeitern wurde etwa 1964 die Kommission der Kirchen für Migranten in Europa (Churches' Commission for Migrants in Europe - CCME) gegründet, die dann 1978 ihren Sitz von Genf nach Brüssel

\footnotetext{
${ }^{23}$ Vgl. hierzu das "Preliminary statement“, zitiert bei Ebd., S. 30.

${ }^{24}$ Ebd., S. 6.
} 
verlegte und zu einer eigenständigen, von anglikanischen, orthodoxen und protestantischen Kirchen getragenen ökumenischen Organisation in Brüssel wurde.

Daneben entstanden andere, protestantisch geprägte Europakreise in Paris und Brüssel. ${ }^{25}$ So kamen bspw. in Brüssel seit 1959 einmal im Monat Beamte aus den verschiedenen Europäischen Institutionen mit ihren Familien zum gemeinsamen Gottesdienst in der evangelischen Kirche in der Rue Belliard in Brüssel zusammen. ${ }^{26}$ Daraus entwickelte sich aus der ungefähr 100 Personen umfassenden, ökumenisch orientierten Gruppe das

Ökumenische Zentrum Brüssel, das sich mit regelmäßigen Veranstaltungen, Vorträgen und Diskussionen mit aktuellen europapolitischen Fragen auseinandersetzte. Es wurde von sechs Pfarrern und zehn Laien geleitet und besaß seit 1959 sogar zwei eigene Studiengruppen, die sich mit der Bedeutung des Menschen in den Europäischen Institutionen sowie mit der Rolle von Kirchen und Christen im sich vollziehenden wirtschaftlichen und politischen Einigungsprozess beschäftigten. ${ }^{27}$ Leitender Kopf dieser Brüsseler Gruppe war bis 1964 der niederländische Baron Carel Diederic Aernaut Baron van Lynden, Generalsekretär der Benelux-Wirtschaftsunion. Er war zunächst von Max Kohnstamm nach Luxemburg geholt worden und teilte mit diesem Erfahrungen aus dem niederländischen Widerstand. ${ }^{28}$ Van Lynden zur Seite stand in Brüssel der Deutsche Helmut von Verschuer, der nach diesem das Ökumenische Zentrum von 1964 bis 1984 ebenfalls ehrenamtlich leitete. In von Verschuer wiederum kreuzten sich die Linien des europäischen Widerstandes, der Ökumenischen Bewegung und der entstehenden EU. Denn von Verschuer, der zunächst Mitglied der ständigen deutschen Delegation für die Verhandlungen über eine europäische Agrarunion und seit 1956 u.a. an den Verhandlungen zum EWG-Vertrag und über eine Europäische Freihandelszone beteiligt war und schließlich seit 1958 in der Europäischen Kommission in der Generaldirektion Landwirtschaft arbeitete, war ein Neffe Adam von Trotts. ${ }^{29}$ Über die Vermittlung seiner Tante Vera von Trott, einer Schwester Adam von Trotts, hatte von Verschuer 1957 Kontakt mit Visser 't Hooft aufgenommen und ihn in Genf besucht. Dort

\footnotetext{
${ }^{25}$ Ausführlicher Ebd., S. $24 \mathrm{f}$.

${ }^{26}$ Vgl. hierzu auch Katharina Kunter, Die Kirchen - Europa - die Ökumene, in: Claudia Lepp/Kurt Nowak (Hg.), Evangelische Kirche im geteilten Deutschland (1945-1989/90), Göttingen 2001, S. 255-276; hier v.a. S. 268.

${ }^{27}$ Vgl. hierzu ausführlicher Win Burton, The European Vision and the Churches. The Legacy of Marc Lenders, Genf 2015.

${ }^{28}$ Ebd, S. 7.

${ }^{29}$ Instruktive Einsichten aus der Frühzeit der Europäischen Kommission und des Brüsseler Ökumenischen Zentrums vermittelt der Zeitzeugenbericht von Helmut Van Verschuer, vgl. Oral History Project - The European Commission 1958-1972, European University Institute 1994-2007; S. 34.
} 
informierte ihn Visser 't Hooft über die CCREC und lud ihn seit dem Herbst 1958 zur Mitarbeit ein. Zudem ergaben sich aus dieser Begegnung in den folgenden Jahren zahlreiche weitere Kontakte und Vernetzungen zwischen der Genfer CCREC-Gruppe, der Brüsseler Gruppe, der KKIA und der entstehenden KEK mit ihren Führungspersönlichkeiten. ${ }^{30}$ Von der Europäischen Kommission erfuhr das Ökumenische Zentrum in Brüssel insbesondere in seinen Anfangsjahren viel Unterstützung, da die leitenden Europabeamten die im Ökumenischen Zentrum zusammenkommenden Christen und Kirchen als ein dem Gemeinwohl verpflichteter Motor und Partner der Europäischen Idee sahen und deshalb gerne zu Vorträgen oder Diskussionen in dieses kamen. ${ }^{31}$ Dabei unterschied sich der von protestantischen Laien getragene Ansatz des Brüsseler Zentrums von dem ebenfalls in Brüssel agierenden katholischen Foyer Catholique Européen, dessen Pfarrer sich insbesondere auf die Seelsorge unter den europäischen, vor allem den italienischen Beamten, konzentrierten. Mit der zunehmenden Institutionalisierung und Professionalisierung der EG wurde im Ökumenischen Zentrum immer deutlicher, dass sich der Beitrag von Christen nicht nur auf das Anbieten einer offenen Gesprächsplattform im Integrationsprozess beschränken konnte, sondern dass es zur eigenen Interessenwahrnehmung auch eine lokale Repräsentation der Kirchen aus den Mitgliedsstaaten der Europäischen Union geben musste. Von Verschuer griff diese Idee auf und präsentierte auf der Gründungsversammlung der KEK 1964 auf der MS Bornholm den Vorschlag eines Ressourcen bündelnden Komitees der Kirchen der EG, das angemessen auf die neuen Herausforderungen der politischen Institutionen Westeuropas eingehen und die verschiedenen christlichen Europakreise in Brüssel besser zusammenfassen könne. Ihm folgte 1964 die Gründung der Kommission der Kirchen bei den Europäischen Gemeinschaften (engl. Consultative Commission of Churches fir the European Communities, CCCEC) in Brüssel, die 1984 in Europäische Ökumenische Kommission für Kirche und Gesellschaft unbenannt wurde (engl. European Ecumenical Commission for Church and Society, EECS). Das Ökumenische Zentrum blieb weiterhin bestehen und diente der neuen Kommission als eine Art Sekretariat, das vor allem für die Kontaktpflege und Vermittlungs- und Koordinierungsaufgaben zuständig war. Dazu wurde 1966 auf Empfehlung Egbert Emmens, dem Generalsekretär der Nederlandse Hervormde Kerk und KEK-Gründungsmitglied, der

\footnotetext{
${ }^{30} \mathrm{Vgl}$. hierzu ausführlicher W. Burton, Lenders.

${ }^{31}$ Vgl. hierzu Interview Van Verschuer, in: Oral History Project - The European Commission 1958-1972, European University Institute 1994-2007.
} 
niederländisch-belgische Pfarrer Marc Lenders als Sekretär des Ökumenischen Zentrums in Brüssel eingestellt. ${ }^{32}$ Über die Zusammenarbeit mit der Kommission hinaus blieb es jedoch bis zu seiner Auflösung im Jahr 1991 nicht nur eine zentrale Anlaufstation für hochrangige Mitglieder der Europäischen Kommission, sondern formte zusammen mit der Kommission eine ökumenische Plattform, die in den Mitgliedskirchen der EU langsam das Bewusstsein schärfte, dass sich auch die evangelischen Kirchen stärker in europäischen Strukturen und gemeinsamen Institutionen organisieren müssten.

\section{Evangelische Distanz gegenüber Westeuropa}

Bei zahlreichen Protestanten und evangelischen Kirchen in Europa blieb jedoch das Engagement, mit dem das Ökumenische Zentrum und die Kommission der Kirchen in Brüssel das Voranschreiten der europäischen Integration unterstützten, unbekannt oder wurde sogar bis in die 1980er Jahre hinein mit Skepsis betrachtet. Insbesondere dem deutschen Protestantismus falle es offensichtlich schwer, Europa mit etwas anderem als der Ost-WestSpaltung zu identifizieren, beobachtete etwa der Schweizer Theologe Lukas Vischer 1979 als Gast auf einem Europa-Podium auf dem Evangelischen Kirchentag in Nürnberg und formulierte:

„(...) wenn ich vor allem den Applaus richtig interpretiert habe, beginnen hier in Deutschland die Seelen zu vibrieren, sobald die Feststellung fällt, daß West und Ost durch eine kulturelle Einheit verbunden seien. Hier werden die Seelen wach.

Westeuropa hingegen wird nicht durch dieselbe emotional gefestigte kulturelle Gemeinsamkeit zusammengehalten.“(Deutscher Evangelischer Kirchentag 1979, S.690) $)^{33}$

Auch in der Kirchenkanzlei der Evangelischen Kirche in Deutschland (EKD) teilte man diese Ansicht und war der Meinung, dass Europa einerseits vor allem durch die Ost-West-Spaltung gekennzeichnet sei und andererseits Westeuropa vor allem eine wirtschaftliche Macht darstelle. ${ }^{34}$ Diese von zahlreichen evangelischen Kreisen in der Bundesrepublik (und auch in der DDR) geteilte Auffassung verkannte allerdings nicht nur die zunehmende Europäisierung

\footnotetext{
${ }^{32}$ Einen ausführlichen Lebensbericht über Lenders bietet W. Burton, Lenders.

${ }^{33}$ In: Europa - Überwindung von Grenzen, Podiumsgespräch am 15.6. in: Deutscher Evangelischer Kirchentag, Nürnberg 1979, Dokumente, hg. i.A. des Präsidiums des Deutschen Evangelischen Kirchentages, Stuttgart 1979, S. 690.

${ }^{34}$ Vgl. Vizepräsident der EKD-Kirchenkanzlei in einem Brief an den Bevollmächtigten des Rates der EKD am Sitz der Bundesregierung, Prälat Heinz Georg Binder, am 28.9.1977, in: Evangelisches Zentralarchiv 2 /93/3794 (ausführlich zitiert in K. Kunter, Die Kirchen - Europa, S. 270).
} 
ehemals partikularstaatlicher Politik und Interessen. Sie hatte darüber hinaus ebenfalls nicht im Blick, dass es sich bei der Entwicklung und Etablierung der EU um die Festigung eines neuen supranationalen Rechtssystems und seiner entsprechenden Einrichtungen handelte und nicht nur um die Errichtung einer weiteren internationalen Organisation in Europa. Lähmend wirkten sich in dieser Hinsicht zusätzlich die Zusammenkünfte und Diskussionen aus, die im Rahmen der KEK zu Europa stattfanden. Auch hier kam eine unüberbrückbare Kluft zwischen „Brüssel und Westeuropa“ auf der einen Seite und der von den KEKRepräsentanten im Großen und Ganzen vertretenen Idee von Europa als einem geistlichkulturellen Paneuropa zum Ausdruck. Sicherlich spielte dabei eine Rolle, dass die KEKMitglieder konfessionsübergreifend sowohl evangelischen, anglikanischen und orthodoxen Kirchen entstammten und in ihrer Mehrzahl aus Pfarrern, ordinierten Theologen und Kirchenvertretern bestanden - und kaum beruflich-fachliche Berührungspunkte mit dem politischen Integrationsprozess hatten. Zudem machte sich der ideologische Einfluss staatssozialistisch geprägter Theologen bemerkbar, die die Europäische Gemeinschaft gerne als wirtschaftliches Äquivalent der NATO abwerteten. Dies war auch der Tenor der Verlautbarungen zur Europäischen Integration der moskauorientierten Christlichen Friedenskonferenz (CFK). Wie schwer es der protestantischen Ökumene - und diese umfasste neben der KEK auch den ÖRK und die konfessionellen Weltbünde, also den Reformierten und Lutherischen Weltbund - fiel, Europa als einen nach vorne zu gestaltenden politischen Prozess zu begreifen, zeigte sich dann deutlich 1972 mit dem Beginn der Konferenz für Sicherheit und Zusammenarbeit (KSZE), die 1975 mit der Schlussakte von Helsinki abgeschlossen wurde. ${ }^{35}$ Nach der anfänglichen Dominanz des Friedensthemas, das vor allem von den osteuropäischen Kirchenvertretern auf die ökumenische Agenda gebracht worden war, setzte sich nach und nach beim ÖRK, bei der KKIA, dem Lutherischen Weltbund und der KEK sowie in den evangelischen Kirchen in der Bundesrepublik und in der DDR die zentrale Frage nach der Geltung und der Durchsetzung der Menschenrechte durch. Trotz der anhaltenden Verletzungen der Religionsfreiheit in den sozialistischen Staaten Mittel- und Osteuropas gab es innerhalb der protestantischen Ökumene Versuche, eine explizit westliche Positionierung in der Menschenrechtsfrage zu vermeiden. Statt dessen wurde erneut nach einem Mittelweg zwischen Ost- und West gesucht. Sowohl in der KEK, als auch

\footnotetext{
${ }^{35}$ Vgl. hierzu ausführlich Katharina Kunter, Die Kirchen im KSZE-Prozess 1968-1978, Stuttgart 2000.
} 
im ÖRK und im deutschen Protestantismus wurde dabei besonders intensiv um die Frage gerungen, ob die Menschenrechte stärker liberal-individualistisch oder sozialistisch-kollektiv zu interpretieren seien. Die den kirchlichen Reflexionsprozess zur KSZE abschließende KEKKonsultation zum Thema „Die KSZE und die Kirchen“, die drei Monate nach der Unterzeichnung der Schlussakte von Helsinki in Buckow in der DDR stattfand, wählte auch hier einen Dritten Weg, als sie zum „Prinzip VII“ der Schlussakte von Helsinki zwar festhielt, dass sie die Aussagen über die Gedanken-, Gewissen-, Religions- und Überzeugungsfreiheit sowie über die Kirchen als besonders wirksam erachte, zugleich aber auf die Unteilbarkeit der zehn Prinzipien hinwies - und sich damit deutlich der sozialistischen Lesart der Schlussakte von Helsinki annäherte. ${ }^{36}$ Auch im Bund der Evangelischen Kirchen in der DDR bevorzugte man die in Buckow formulierte Kompromissformel und hielt fest, dass Menschenrechte in ihren jeweiligen sozialen Kontext zu verwirklichen seien. ${ }^{37}$ Es verwunderte vor diesem Hintergrund nicht, dass erst die von KPdSU-Generalsekretär Michail Gorbatschow im Kontext von Perestroika und Glasnost skizzierte Vision vom gemeinsamen europäischen Haus die protestantische Ökumene zu einem breiteren Europaengagement mobilisierte. Eingebettet in den sogenannten konziliaren Prozess für Frieden, Gerechtigkeit und Bewahrung der Schöpfung, der offiziell 1983 auf der Vollversammlung des ÖRK in Gang gebracht worden war ${ }^{38}$, war es vor allem die im Mai 1987 gemeinsam von der KEK und dem Rat der Europäischen Bischofskonferenzen (CCEE) ausgerichtete Erste Europäische Ökumenische Versammlung in Basel, die als dem ersten europaweiten Kirchentreffen seit 1431 mit über 700 Delegierten aus sämtlichen Konfessionen Europas einen eigenen Beitrag zur Europäischen Einheit leisten wollte. Doch auch hier dominierte in den Vorbereitungsgruppen auf protestantischer Seite einerseits das Unvermögen, überhaupt ein gemeinsames europäisches Anliegen für die Kirchen ausmachen zu können, und andererseits das Bemühen, durch eine kirchliche Mittelposition die sich abzeichnende westeuropäische Dominanz der Teilnehmenden sowie der von ihnen vertretenen Wirtschafts- und Gesellschaftskonzepte zu durchbrechen. ${ }^{39}$ So mahnte etwa der Erfurter Probst Heino Falcke, dass Basel eine gesamteuropäische Konferenz werden müsse,

\footnotetext{
${ }^{36}$ Vgl. K. Kunter, KSZE, S. 145.

${ }^{37}$ Auch die EKD tat sich mit einer westlichen Positionierung im Hinblick auf die Menschenrechte zu dieser Zeit schwer, vgl. die Thesenreihe „Zur Frage der Menschenrechte“ vom 30.8.1974 sowie der EKD-Stellungnahme „Die Menschenrechte im ökumenischen Gespräch“ vom 26.9.1975.

${ }^{38} \mathrm{Vgl}$. hierzu ausführlich Katharina Kunter, Erfüllte Hoffnungen und zerbrochene Träume. Evangelische Kirchen in Deutschland im Spannungsfeld von Demokratie und Sozialismus (1980-1993), Göttingen 2006.

${ }^{39}$ Vgl. Ebd., S. 53.
} 
und dementsprechend auch die Rolle des Sozialismus in Europa und die neuen Hoffnungen auf eine Perestroika im Gegenüber zu Westeuropa und seinen kapitalistischen Staaten thematisiert werden sollten. Basel wurde, trotz einer fehlenden Europakonzeption und der nach wie vor zum Ausdruck gebrachten Distanz gegenüber dem westeuropäischen Integrationsprozess, zu einem Erfolg, was insbesondere auf den bereits spürbaren osteuropäischen Aufbruch einer sich findenden Zivilgesellschaft zurückzuführen war.

\section{Protestantische Neuorientierungen nach 1990}

Mit dem Fall des Eisernen Vorhangs und dem Ende der sozialistischen Regierungen in Mittelund Osteuropa tat sich die Genfer Ökumene schwer. Die Weltkonferenz von Seoul 1990 fand keine positiven und zustimmenden Worte zum Ende des Sozialismus und zum Einsatz der teils auch christlich motivierten Bürgerrechtsbewegungen in Mittel- und Osteuropa. Die KEK, die es wie die anderen protestantischen ökumenischen Organisationen versäumt hatte, mit den christlichen Dissidenten in einen Dialog zu kommen, erlitt in der Folgezeit einen großen Vertrauensverlust in Osteuropa. Dieser, der 1993 in Kraft getretene Europäische Binnenmarkt, die 2004 vollzogene EU-Osterweiterung sowie die in diesen Prozessen zunehmend wichtiger werdende kirchliche Interessenvertretung in Europa, vor allem in Brüssel, ebneten dann allerdings nach 1990 grundlegenden Umstrukturierungen den Weg. Das betraf vor allem die alte Struktur und Rolle der KEK, die sich nun stärker Themen und Feldern der EU öffnete und sich nun auch mit ihren Büros in Brüssel und Straßburg positionierte. So schlossen sich 1999 die KEK und die Europäische Ökumenische Kommission für Kirche und Gesellschaft (EECCS) zu einer gemeinsamen neuen Kommission für Kirche und Gesellschaft (engl. Commission for Church and Society of the CEC) mit einer eigenen Repräsentanz in Brüssel und Straßburg zusammen. Auch die Kommission der Kirchen für Migranten in Europa (CCME) wurde in die neue KEK-Struktur integriert und ist nun in Brüssel präsent. Der 1990 von Jacques Delors, dem damaligen Präsidenten der Europäischen Kommission lancierte Aufruf an die christlichen Kirchen in Europa, sich mit an der Suche nach Herz und Seele Europas („Heart and Seoul of Europe“) zu beteiligen, wirkte da einerseits durchaus als willkommener metaphysischer Katalysator, leitete aber andererseits durchaus eine neue Phase kirchenleitenden Europaengagements ein, wie nicht nur die Veränderungen bei der KEK zeigen, sondern etwa auch das 1990 eingerichtete Europabüro der EKD in Brüssel. Inwiefern freilich die Akzeptanz der EU als einem längst vollzogenen 
europäischen supranationalen Rechts-, Gesellschafts- und Wirtschaftssystem, das auf Heterogenität, Wertepluralismus, Rechtssicherheit und Demokratie beruht und in dem christliche Werte daher nicht mehr automatisch als verbindlich angesehen werden, sich dadurch auch in den Breiten des deutschen und europäischen Protestantismus verwurzelt hat, bleibt abzuwarten.

\section{Literaturverzeichnis}

Amsterdamer Dokumente (1949): Berichte und Reden auf der Weltkirchenkonferenz in Amsterdam 1948,2 . Auflage, Bielefeld.

Barth, Karl (1946): Die christliche Verkündigung im heutigen Europa, München.

Brakelmann, Günter (1981): Kirche und Schuld: Das Darmstädter Wort von 1947, in: Ders., Kirche in den Konflikten ihrer Zeit, München.

Burton, Win (2015): The European Vision and the Churches. The Legacy of Marc Lenders, Genf.

Coupland, Philip M. (2006): Britannia, Europa and Christendom. British Christians and European Integration, London.

Garstecki, Joachim (Hrsg.) (2007): Die Ökumene und der Widerstand gegen Diktaturen. Nationalsozialismus und Kommunismus als Herausforderung an die Kirchen, Stuttgart.

Greschat, Martin (1985): Im Zeichen der Schuld, München.

Greschat, Martin (1994): Der Protestantismus und die Entstehung der Europäischen Gemeinschaft, in: Greschat, Martin / Loth, Wilfried (Hrsg.): Die Christen und die Entstehung der Europäischen Gemeinschaft, Stuttgart, S. 25-S.96.

Greschat, Martin / Loth, Wilfried (Hrsg.)(1994): Die Christen und die Entstehung der Europäischen Gemeinschaft, Stuttgart.

Greschat, Martin (2000): Ökumenisches Handeln der Kirchen in den Zeiten des Kalten Krieges, in: Ökumenische Rundschau, Jg. 2000, H. 49, S. 7-25.

Immerman, Richard H. (1998): John Forster Dulles, Piety, Pragmatism, and Power in U.S. Foreign Policy, Washington.

Kohlbrugge, Hebe (2003): Zwei mal zwei ist fünf. Mein unberechenbares Leben seit 1914, Leipzig.

Kohnstamm, Max (1985): Die alten, bösen Lieder. Das Hin und Her um die Vorbereitungen der Reagan-Reise öffnet schon verheilte Wunden, in: Die Zeit Nr. 18 vom 26. April, S.3.

Kunter, Katharina (2000): Die Kirchen im KSZE-Prozess 1968-1978, Stuttgart. 
Kunter, Katharina (2001): Die Kirchen - Europa - die Ökumene, in: Lepp, Claudia / Nowak, Kurt (Hrsg.): Evangelische Kirche im geteilten Deutschland (1945-1989/90), Göttingen, S. 255-276.

Kunter, Katharina (2006): Erfüllte Hoffnungen und zerbrochene Träume. Evangelische Kirchen in Deutschland im Spannungsfeld von Demokratie und Sozialismus (1980-1993), Göttingen.

Kunze, Rolf-Ulrich (2007): Die ganze Kirche für die ganze Welt: Willem Adolf Visser 't Hooft und der Widerstand gegen den Nationalsozialismus, 1933-1945, in: Garstecki, Joachim (Hrsg.), Die Ökumene und der Widerstand gegen Diktaturen. Nationalsozialismus und Kommunismus als Herausforderung an die Kirchen, Stuttgart, S. 32-46.

Leustean, Lucian N. (2014): The Ecumenical Movement and the Making oft he European Community, Oxford.

Lipgens, Walter (Hrsg.) (1968), Europa-Föderationspläne der Widerstandsbewegung 1940-1945, München.

Lipgens, Walter (Hrsg.) (1986): Documents on the History of European Integration, Bd. 2, Berlin / New York.

Mak, Geert (2010): Niederlande, München.

Nurser, John S. (2005): For all Peoples and All Nations: The Ecumenical Church and Human Rights, Georgetown.

Patijn, Cornelis L. (1989): Die „verantwortliche Gesellschaft". Ein Rückblick, in: Ökumenischer Rat der Kirchen (Hrsg.): Es begann in Amsterdam. 40 Jahre Ökumenischer Rat der Kirchen, Frankfurt a.M., S. 62-66.

Pistone, Sergio (2008): The Union of European Federalists, Mailand.

Podiumsgespräch am 15.6.1979 in: Europa - Überwindung von Grenzen, in: Deutscher Evangelischer Kirchentag, Nürnberg 1979, Dokumente, hrsg. i.A. des Präsidiums des Deutschen Evangelischen Kirchentages, Stuttgart.

Oral History Project (1994-2007): The European Commission 1958-1973: History and Memories of an Institution, European University Institute 2007.

Sauer, Thomas (1999): Westorientierung im deutschen Protestantismus? Vorstellungen und Tätigkeit des Kronberger Kreises, München.

Soutou, George-Henri (2014): Amt und Verantwortung: Diplomaten in Deutschland und Frankreich 1933-1945, in: Hürter, Johannes / Mayer, Michael (Hrsg.), Das Auswärtige Amt in der NSDiktatur, Berlin, S. 59-S.74.

Visser 't Hooft, Willem A. (1973): Memoirs, Genf. 
Visser 't Hooft, Willem A. / Oldham, Joseph H. (1937): The Church and Its Function in Society, Chicago.

Wielenga, Friso (2008): Rezension zu Anjo G. Harryvan/Jan van der Harst, Max Kohnstamm. Leven en werk van een Europeaan, Utrecht 2008, Westfälische Wilhelms-Universität Müsnter, erstellt im Mai 2008, https://www.uni-muenster.de/NiederlandeNet/nlwissen/geschichte/vertiefung/europa/kohnstamm.html.

Zeilstra, Jurjen A. (1995): European Unity in Ecumenical Thinking 1937-1948, Zoetermeer. 